\title{
Einzelprojekt „Wellen“
}

\author{
Andreas Witzig
}

Wellenphänomene werden in den Lehrplänen meist erst nach gründlicher Vorbereitung der formalen Grundlagen eingeführt. Die Studierenden verfügen dann über ein mathematisches Rüstzeug, dass ihnen den Zugang über analytische Formeln erlaubt. Als Alternative zu diesem mathematiklastigen Weg möchten wir in diesem Projekt das Experimentieren mit Computersimulationen ins Zentrum stellen. Anhand von zwei konkreten physikalischen Gesetzen, der Ausbreitung elektromagnetischer Wellen und dem Verhalten von quantenmechanischen Teilchen, haben wir Lernmaterialien erarbeitet, die einen intuitiven Zugang zum Thema Wellen unterstützen und eine Brücke schlagen zwischen Theorie und der Anschauung. Die Materialien wurden mit Ingenieurstudierenden im Studiengang Systemtechnik an der School of Engineering der Zürcher Hochschule für angewandte Wissenshaften getestet und verbessert.

Wir sind überzeugt, dass der Einsatz von Computersimulationen den Physikunterricht bereichert und die Studierenden aktiviert. Die eingesetzten Tools bieten hervorragende Visualisierungen und laden ein zum Experimentieren. Softwarewerkzeuge haben außerdem ein hohes Potenzial für den Einsatz im Fernunterricht und im orts- und zeitunabhängigen Lernen.

Physikunterricht soll die kognitive Wahrnehmung erweitern. Wir haben beobachtet, wie sich ein tiefergehendes Verständnis von Wellenphänomenen bildet. Dabei ist aufgefallen, dass die Studierenden unterschiedlich weit entwickelt sind und dass geeignete Visualisierungstools die Erweiterung der Vorstellungskraft gut unterstützen. Thematisch knüpft der Unterricht an vorhergehende Veranstaltungen

\footnotetext{
A. Witzig $(\bowtie)$

Institute of Computational Physics, ZHAW School of Engineering, Winterthur, Schweiz E-Mail: andreas.witzig@zhaw.ch
} 
an und schlägt eine Brücke auf die spätere Verwendung des Stoffes - zwei natürliche Nahtstellen, die im Seamless Learning Kontext herausgearbeitet wurden. Das Vorwissen als Basis für das Verständnis der Lerninhalte und die Referenz auf den Ingenieur-Berufsalltag sind wichtige motivierende Elemente in der Vorlesung.

Die Anforderungen an die Didaktik-Simulation unterscheidet sich stark von numerischen Lösungsverfahren, die in Forschung und Entwicklung bzw. in der Industrie eingesetzt werden. Wenn als essentielle Schnittstelle der Übergang aus dem Ingenieurstudium in den Berufsalltag diskutiert wird, so stellt sich dabei ein Dilemma: einerseits möchte man didaktisch optimierte Tools einsetzen, andererseits wäre es schön, wenn die Studierenden die Simulationswerkzeuge ihrer späteren Berufspraxis kennenlernen würden. Im Themenbereich der elektromagnetischen Wellen haben wir sowohl ein reines Didaktik-Tool als auch ein kommerzielles Ingenieurtool eingesetzt und die Unterschiede analysiert.

\subsection{Ausgangssituation}

Im Physikunterricht soll der Graben überwunden werden zwischen der formalen Repräsentation, also den physikalischen Grundgleichungen, und dem konkret für die Studierenden zugänglichen und erlebbaren Realität. Das Material wird den Studierenden mit dem Ziel vermittelt, dass sie es später in ihrer Tätigkeit in einer Ingenieurdisziplin wieder zur Verfügung haben. Diese spätere Anwendung umfasst in der Regel nicht den technischen Entwurf oder die vertiefte Forschungstätigkeit mithilfe der Wellentheorie. Vielmehr wird davon ausgegangen, dass in einem komplexen Zusammenhang im Ingenieuralltag Fragestellungen beurteilt werden müssen, die auf der Vorstellungskraft von Wellenphänomenen beruhen. Der formale mathematische Umgang mit den Grundgleichungen wird mit großer Wahrscheinlichkeit im weiteren Bildungs- und Berufsweg unserer Studierenden gar nicht mehr gefordert. Wir suchen deshalb nach didaktischen Konzepten, die die Anschauung trainieren und weniger die analytischen Umformungen der Gleichungen.

Für den Unterricht ergibt sich daraus die Herausforderung, dass aufschlussreiche Bilder portiert werden, die den Studierenden auch Jahre nach Studienabschluss in Erinnerung bleiben. Es sollen Fertigkeiten geübt werden, die über das Lösen von Übungsaufgaben hinausgehen. Es scheint uns realistisch und akzeptabel, dass Ingenieure die mathematischen Konzepte einige Zeit nach ihrem Bachelor-Abschluss nicht vollständig wiedergeben können. Ein praxisorientierter Unterricht soll sie dazu befähigen, später einmal kreativ und lösungsorientiert 
Entscheide im Zusammenhang mit dem Entwurf oder mit der Einschätzung von Effekten treffen zu können.

\section{Experimente im Physikunterricht}

Oft helfen real aufgebaute Experimente, wie sie in der Tradition der School of Engineering im Physikunterricht regelmäßig zum Einsatz kommen. Die Sammlung der Fachhochschule umfasst über 800 Experimente und wird sorgfältig gepflegt. Die Ingenieurschule investiert viel in den Unterhalt der Exponate und stellt ein digitales Verwaltungs- und Bestellsystem zur Verfügung. Die Physikwerkstatt und die Dozierenden sorgen dafür, dass Sammlung und Datenbank lebendig bleiben, dass die Versuche funktionieren und dass ergänzende Unterrichtsmaterialien und Tipps und Tricks für die Durchführung unter den Lehrenden rege ausgetauscht werden. Diese Voraussetzungen sind eine wichtige Grundlage für das vorliegende Projekt, da einerseits an der Schule eine Tradition zum langfristigen Aufbau und dem Austausch von Unterrichtsmaterialien besteht. Andererseits sind die vorgeschlagenen digitalen Experimente immer als Ergänzung zu (und nicht etwa als Ersatz von) den Exponaten der realen Welt zu verstehen.

Im Physikunterricht werden schon seit über 40 Jahren auch Computersimulationen eingesetzt (Boardman et al., 1989), wobei die verwendeten Softwaretools und Unterrichtsmaterialien über die Zeit stark verbessert wurden (Finkelstein, 2005; Perkins et al., 2006).

Erst seit wenigen Jahren bringen alle Studierenden leistungsfähige mobile elektronische Geräte in den Unterricht. Für den Einsatz von Simulationstools in Übungen und Praktika ist dies eine große Chance.

Als einer der wichtigsten Aspekte des Seamless Learning wird im Zusammenhang mit Physikexperimenten das Zusammenspiel von realen, analogen Experimenten mit den neuen digitalen Ressourcen gesehen. Wir erkennen eine gegenseitige Befruchtung von Hardware-Exponaten und Computersimulation, und die Resultate des vorliegenden Projektes müssen im Kontext des Präsenzunterrichts gesehen werden. Die bewusste Wahl „Analog oder Digital“ sowie die Möglichkeit zum Wechsel des Mediums sind ein Mehrwert für die Lehre. 


\section{Modulbeschreibung und Lernziele}

Die in diesem Projekt geschaffenen Lernobjekte sollen im 4-ECTS-Modul „Physik: Felder und Wellen“ bzw. „Physik 3“ im zweiten Studienjahr des Studiengangs „Systemtechnik“ an der School of Engineering der Zürcher Hochschule für Angewandte Wissenschaften ZHAW eingesetzt werden. Aus Sicht der Studierenden umfasst das Modul während 14 Wochen zwei Lektionen Vorlesung und zwei Lektionen Übung/Praktikum in Halbklassen, plus Hausaufgaben während dem Semester und einer Vorbereitungszeit auf die Semesterendprüfung.

Die Modulbeschreibung, die in diesem Projekt allerdings nicht beeinflusst werden konnte, umfasst folgende inhaltliche Zusammenfassung und die Lernziele:

- Ausgehend von mechanischen Wellen wird die mathematische und physikalische Beschreibung entwickelt und auf elektromagnetische Wellen ausgedehnt. Elektromagnetische Phänomene werden auf die Maxwellgleichungen zurückgeführt. Im Anschluss werden die verschiedenen Zugänge zur Optik beleuchtet. Im zweiten Teil der Vorlesung wird die quantenphysikalische (wellenmechanische) Beschreibung von Teilchen und die Interaktion von Licht mit Materie behandelt.

- Lernziel 1: Studierende können für die Systemtechnik relevante Wellenphänomene (mechanisch/Schall und elektromagnetisch/Radiowellen, Infrarot, Licht) qualitativ und quantitativ beschreiben und deren Implikationen für technische Anwendungen nennen.

- Lernziel 2: Studierende sind in der Lage, einfache nummerische Methoden und Computersimulationen für die Untersuchung und Analyse von Wellen oder Wellenphänomenen zu verstehen und für technische Anwendungen einzusetzen.

- Lernziel 3: Studierende können die grundlegenden elektromagnetischen Gesetze (Felder, Ladungstransport, Induktion) auf die Maxwellgleichungen zurückführen.

- Lernziel 4: Studierende können zur Beschreibung optischer oder atom/kernphysikalischer Phänomene die geeignete Beschreibungsebene (Strahl, Welle, Teilchen) wählen. Sie kennen die wichtigsten physikalischen Konzepte der Optik, Quanten- und Kernphysik, welche für das Verständnis der Module der Vertiefung Medizintechnik und des Profils Photonics notwendig sind. 
- Modulinhalte: Mechanische Wellen (Transversal und Longitudinalwellen, Schallwellen), stehende Wellen, Intervalle und Partialschwingungen, Dispersion, Reflektion und Impedanz-Sprünge, Dopplereffekt, Schallpegel. Elektromagnetische Feldgleichungen, elektromagnetische Wellen. Optik: geometrische Optik (Refraktion, Linsen, para-axiale Optik), Wellenoptik (Beugung, Polarisation). Einführung in die Quanten- und Atomphysik: Axiome/Grundlagen der Wellenmechanik, Tunneleffekt, Teilchen im Potentialtopf, Energieniveaus in der Elektronenhülle, Halbleiter, Photodioden, Leuchtdioden und Laser (als Anwendungen). Kerne und Teilchen: Aufbau Atomkerne, Nuklidkarte, Radioaktiver Zerfall.

Die Vorlesung wurde in der Vergangenheit in enger Abstimmung mit dem Studiengang Elektrotechnik gehalten, wird derzeit aber aufgrund eines neuen Studienmodells überarbeitet und spezifisch auf die Bedürfnisse des Studiengangs Systemtechnik ausgerichtet.

\section{Umstellung auf Online-Unterricht aufgrund der Covid19-Pandemie}

Einige Rahmenbedingungen haben sich im letzten Projektjahr grundsätzlich verändert, als aufgrund der Covid19-Pandemie der Unterricht in kurzer Zeit auf Fernunterricht umgestellt werden musste. Diese Herausforderungen waren zwar im Projektantrag nicht vorgehen, konnten aber zusätzlich zu den geplanten Fragestellungen bearbeitet werden. Die Umstellung zwischen dem Vor-Ort-Unterricht im Hörsaal und dem zwingend über elektronische Medien durchgeführten Unterricht kann genauso im Kontext des Seamless Learning gesehen werden, wie andere Projektfragestellungen.

In anderen Jahren wurde in der zweiten Hälfte des Semesters eine Zwischenprüfung angeboten. Im Frühlingssemester 2020 wurde aufgrund der HomeOffice-Vorgaben darauf verzichtet und anstelle der Zwischenprüfung eine größere Hausarbeit ausgegeben. Es gab vier Aufgabenstellungen, in welchen mit Anwendung von Computersimulation ein physikalisches Problem gelöst werden musste. Der Arbeitsauftrag war fakultativ und zählt mit $20 \%$ Notengewicht verbessernd zur Semesterendnote. Das neue Format wurde in das Didaktikkonzept der Gesamtveranstaltung übernommen, hat sich bewährt und wird in Zukunft weitergeführt. 


\subsection{Lernobjekte}

Für beide Themenbereiche elektromagnetische Wellenausbreitung und Quantenmechanik werden Simulationen ins Zentrum der Lernerfahrung gestellt. Während die Phänomene der Elektromagnetischen Felder auch viele gute Experimente existieren, sind Labormessungen in der Quantenmechanik viel komplizierter und der Stellenwert von Simulationen deshalb dort höher. Wir setzen ein für die didaktische Visualisierung spezialisiertes Werkzeug ein, das Vorgänge wie die „Quantenmessung“ oder Phänomene wie den „Welle-Teilchen Dualismus“ besonders gut darstellt. Die Software wurde im Rahmen des vorliegenden Projektes erweitert und veröffentlicht.

Die Lernobjekte werden in kleinen Einheiten organisiert, sodass die Chance grösser ist, dass sie in anderen Veranstaltungen ebenso eingesetzt werden können.

\section{Numerische Experimente mit elektromagnetischen Wellen in 2D und 3D}

Die räumliche Ausbreitung von Radio- und Mikrowellen, sowie auch von Wärmestrahlung und Licht zeigt die Natur der Wellenausbreitung und findet sich in vielen wichtigen Anwendungen aus der Technik wieder, beispielsweise in der Signalübermittlung über eine Sender- und eine Empfängerantenne.

Während in vielen Physikbüchern die formelmäßig Betrachtung gleich zu Beginn auf den harmonischen Fall eingeschränkt wird, also auf sinusförmige Anregungen, haben wir hier bewusst die zeitabhängige Beschreibung gewählt, d. h. die physikalischen Größen sind Funktionen der Zeitvariable $t$ :

$$
\begin{gathered}
\operatorname{rot} \vec{E}=-\mu_{0} \frac{\partial \vec{H}}{\partial t} \\
\operatorname{rot} \vec{H}=\vec{j}_{q}(\vec{r}, t)+\sigma(\vec{r}) \vec{E}+\varepsilon(\vec{r}) \frac{\partial \vec{E}}{\partial t}
\end{gathered}
$$

Die gekoppelten Gleichungen sind ein Teil der Maxwellgleichungen, was im Unterricht als Anknüpfung an die traditionelle Beschreibung der Physik ein wichtiger Ausgangspunkt ist. Die Gleichungen zeigen die wichtige gegenseitige Beeinflussung des elektrischen Feldes $\vec{E}(\vec{r}, t)$ und des Magnetfeldes $\vec{H}(\vec{r}, t)$, beide mit einer mehrdimensionalen Ortsabhängigkeit $\vec{r}$ und abhängig von der Zeit $t$. Da die meisten Materialien keine Magnetisierung aufweisen, wird die 
magnetische Permeabilität $\mu=\mu_{0}$ im ganzen Raum konstant angenommen. Die Permittivität $\varepsilon(\vec{r})$ und die elektrische Leitfähigkeit $\sigma(\vec{r})$ hingegen werden als räumlich abhängige Grössen in der Formel belassen. Der Strom $\vec{j}_{q}(\vec{r}, t)$, ebenfalls eine zeitlich variierende Größe, wird als Quellterm separat aufgeführt. Meist ist er überall im Raum Null außer am Ort der Quelle.

Der Übergang von der Integraldarstellung zur Differentialform wird in der Vorlesung aufgezeigt, und die örtlichen Ableitungen Rotation $\operatorname{rot}(\vec{v})$, Diver$\operatorname{genz} \operatorname{div}(\vec{v})$ und Gradient $\operatorname{grad}(s)$ werden aus physikalischer Sicht eingehend diskutiert. Sowohl Integralrechnung als auch Differentialoperatoren sind im vorangehenden Mathematikunterricht eingeführt worden.

Auf weitere Umformungen der Gleichungen (4.1) und (4.2) wird dann absichtlich - und in Abweichung zu vielen Physikbüchern und Vorlesungen - verzichtet. Traditionell würden in der weiteren Argumentation viele Vereinfachungen gemacht: Es würde ein homogener unendlicher Raum angenommen und die Betrachtung auf eindimensionale Fälle beschränkt. Weiter würde man durch analytische Umformungen vom Zeitbereich in den Frequenzbereich wechseln. Damit man noch Lösungsfunktionen für die Gleichung angeben kann, sind diese Vereinfachungen nötig. Man verliert dabei aber die direkte Umsetzung auf relevante Anwendungsfälle.

Alternativ werden im Lernobjekt ,Wellen“ die Gleichungen (4.1) und (4.2) durch einen numerischen Lösungsalgorithmus im Zeitbereich gelöst, wie sie seit über 50 Jahren bekannt sind (Yee, 1966). In einer ersten Version wurde ein auf Matlab ${ }^{1}$ basierender Algorithmus eingesetzt, basierend auf einem öffentlich verfügbaren Skript (Kozhevnikov, 2014), das für den Unterricht entsprechend angepasst wurde. Einerseits wurde mit Matlab ein wichtiges, universelles Ingenieurtool gewählt, was von den Studierenden grundsätzlich positiv aufgenommen wird. Andererseits war die Evaluationszeit mit einigen Minuten CPU-Zeit relativ langsam, sodass das interaktive Element im Praktikum etwas zu kurz gekommen ist. Bei der Überarbeitung des Lernobjekts wurde auf ein browserbasiertes und für didaktische Zwecke optimiertes Tool umgestellt.

Im Vergleich zum allgemeinen Fall der gekoppelten Gleichungen (4.1) und (4.2) wurden für die Simulationen in beiden Fällen als Vereinfachung der dreidimensionale Raum auf 2D reduziert. Die Resultate können auf dem Bildschirm in zwei Dimensionen viel besser betrachtet werden als im allgemeinen 3D-Fall. Außerdem erlaubt die Vereinfachung, dass auch auf handelsüblichen Laptops die Rechenzeit so kurz wird, dass ein echtes Experimentieren durch die Studierenden möglich wird.

\footnotetext{
${ }^{1}$ www.mathworks.com MATLAB (Version R2020b).
} 
Die Darstellung in Abb. 4.1 zeigt eine Illustration, welche den Wellencharakter von Licht der gewohnten Darstellung von Lichtstrahlen gegenüberstellt. Die Studierenden sollen lernen, dass Licht und Wärmestrahlung elektromagnetische Wellen sind und dass ihre Ausbreitung durch die Formeln (1) und (2) beschrieben wird. Im Simulationspraktikum wird dazu ein experimenteller, aktiver Zugang angeboten. Konkret wählen die Studierenden eine willkürliche 2D-Geometrie, indem sie aus Rechtecken und Dreiecken eine Struktur mit hohem Brechungsindex zeichnen. Dann lassen sie eine Lichtwelle auf diese Struktur einfallen und zeichnen den Strahlenverlauf nach. Der Strahlenverlauf ist nur beim Einschalten der Quelle einfach identifizierbar, da nach einiger Zeit wegen den Mehrfachreflexionen störende Interferenzmuster entstehen.

\section{Leitungsgebundene Ausbreitung elektromagnetischer Wellen}

In vielen praktischen Anwendungen werden Signale als elektromagnetische Wellen entlang von Leitungen geführt. Beispiele dafür sind das Koaxialkabel und die Zweidrahtleitungen für elektrische Signale, aber auch die Glasfaser für optische Signale. In einem weiteren Lernobjekt wurden diese leitungsgebundenen Wellen untersucht. Die Simulationen wurden von den Studierenden auf dem Simulationstool Tina ${ }^{2}$ durchgeführt.

Da das Simulationstool für die Schaltungsanalyse bereits früher in der Vorlesung eingeführt wurde (Einschaltvorgänge für Schaltungen mit Widerständen und Kapazitäten, sowie Schaltungen mit Widerständen und Induktivitäten), konnte die Wellenausbreitung als größere Hausaufgabe ausgegeben werden. Die Fragestellung ist im Folgenden wiedergegeben:

Gegeben ist eine lange Übertragungsleitung, zum Beispiel eine Zweidrahtleitung oder ein Koaxialkabel. Simulieren Sie eine solche Leitung mit einer größeren Anzahl von Induktiven und Kapazitiven Abschnitten als Ersatzschaltung in der Simulationssoftware Tina. Als Quelle dient eine Spannungsquelle mit 50 Ohm Innenwiderstand, die einen Puls aussendet. Bei einer geeigneten Dimensionierung der Leitungslänge und einer korrekten Wahl der L- und C-Elemente kann man die Wellenfortpflanzung entlang der Übertragungsleitung visualisieren. Beim Empfänger am Ende der Übertragungsleitung können je nachdem Reflexionen auftreten, die man ebenfalls im Schaltungssimulator abbilden kann.

Zeigen Sie in einer Graphik mit einer Zeitachse auf, wie das Signal an verschiedenen Stellen auf der Leitung aussieht (Strom und Spannung). Kreieren Sie ein bewegtes

${ }^{2}$ https://github.com/pfalstad/ripplegl 


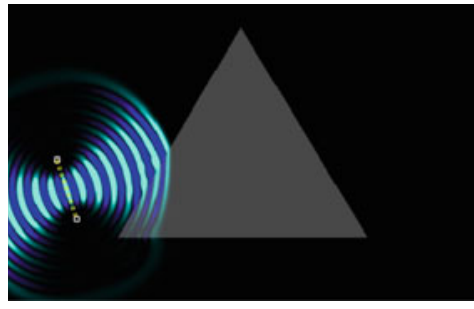

a) Linienquelle (gelb) mit Einschaltvorgang

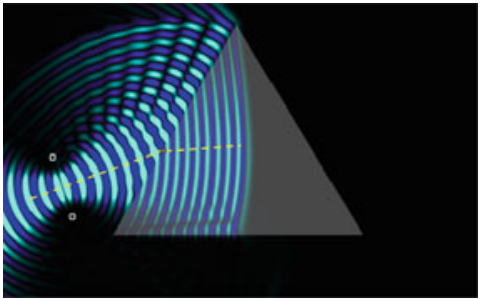

b) Wellenausbreitung senkrecht zur Linienquelle

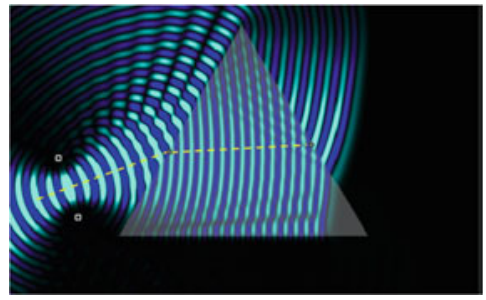

c) Langsamere Ausbreitung im Prisma

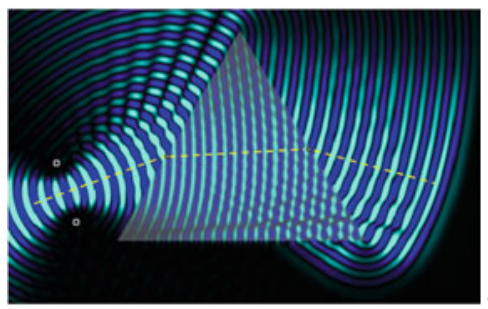

d) Ausbreitung senkrecht zu Wellenfronten

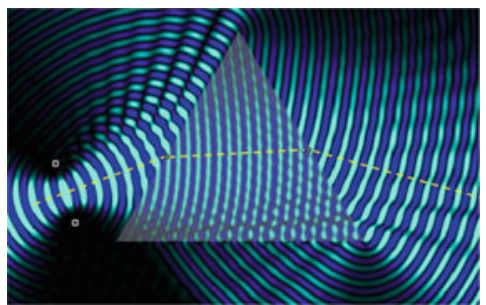

e) Interferenzmuster innen wie außen

Abb.4.1 2D Wellensimulation: Lichtwellen streuen an einem Prisma 
Bild, in dem die örtliche Wellenausbreitung und die Reflexion am Ende der Leitung sichtbar wird.

Die Aufgaben wurden in Zweierteams bearbeitet und wurden durch Simulationen gelöst, wie sie in Abb. 4.2 dargestellt sind. Anstelle einer Schlussbesprechung
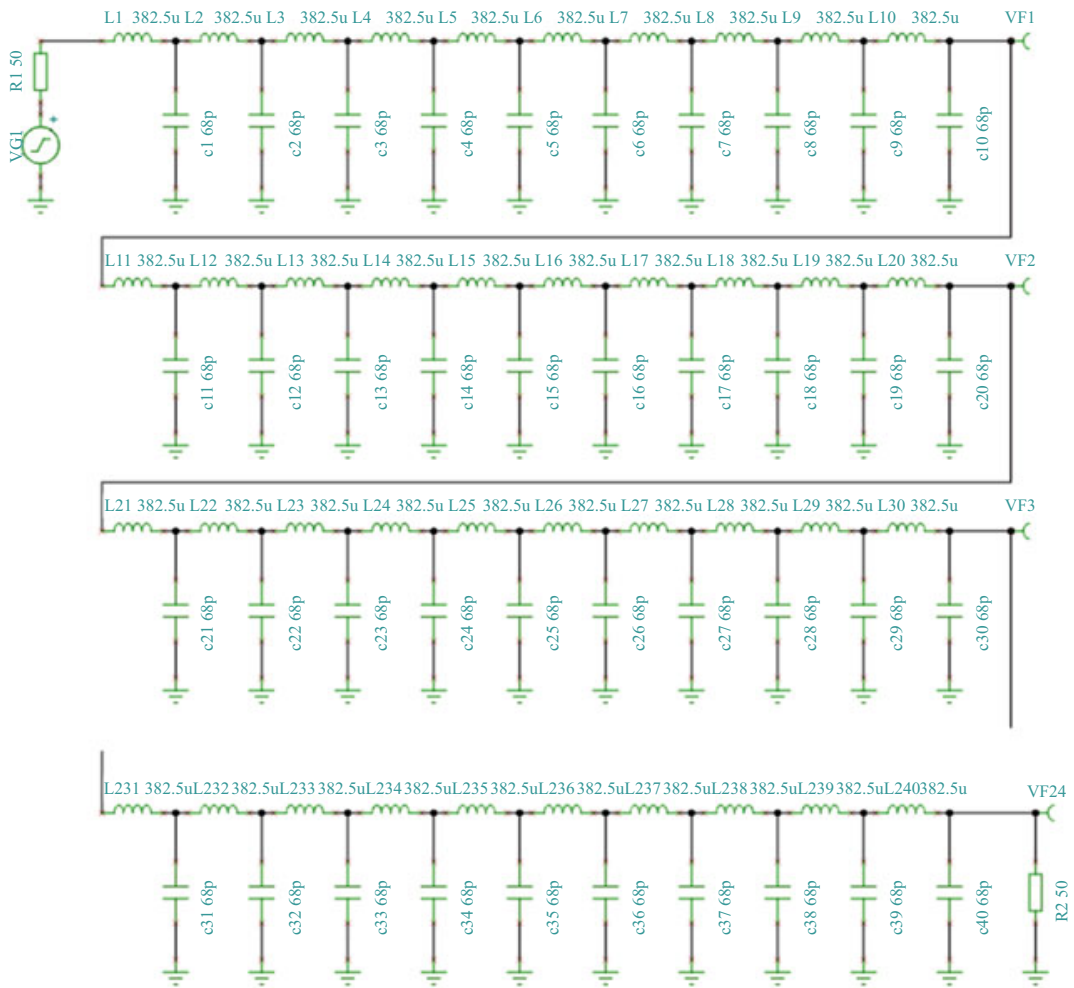

Abb. 4.2 Simulation der leitungsgebundenen Wellenausbreitung. Die Leitung wird durch Induktivitäten $L_{i}$ und Kapazitäten $C_{i}$ dargestellt. Die Quelle hat einen Innenwiderstand von $50 \Omega$. Wenn $L_{i}$ und $C_{i}$ korrekt gewählt werden und der Abschlusswiderstand ebenfalls $50 \Omega$ beträgt, wird die Welle nicht reflektiert. Andernfalls kann beobachtet werden, wie die Welle auf der Leitung mehrmals hin und her läuft. Damit die Wellenausbreitung gut visualisiert werden kann, braucht es eine große Anzahl $L_{i}$ und $C_{i}$. Die gestrichelte Linie deutet an, dass in der Darstellung nicht die ganze Schaltung abgebildet ist 
(nach Bewertung) wurde eine Zwischenbesprechung zwei Wochen vor dem Abgabetermin angeboten. Dieser Termin wurde rege genutzt und war sehr produktiv. Von allen Aktivitäten dieses Projektes waren dies die fortgeschrittensten in Bezug auf das Ingenieurverständnis der physikalischen Problematik. Die Darstellung der Leitung mit einer großen Anzahl von konzentrierten Elementen hat auch die Effekte der örtlichen und zeitlichen Diskretisierung der Simulation offengelegt. Auf der Lernplattform wurde noch folgender Text nachgeschoben, der eine gute Wahl des Pulses vorschlägt:

Bei der Besprechung der Zwischenresultate haben wir gesehen, dass eine RechteckPuls-Anregung in Tina problematisch sein kann. Grund dafür sind die hohen Frequenzanteile, die durch die steilen Flanken verursacht wird. Es ist deshalb empfohlen, die Welle mit einem sanfteren Signal auszulösen (File smoothpulse.pwl). Überlegen Sie sich, wie sie die Zeitachse des ,smoothpulse“ anpassen müssen, damit Sie aussagekräftige Grafiken produzieren können

\section{Wellenausbreitung in der Akustik und auf Wasseroberflächen}

Um die Brücke zu schlagen zu den Beispielen, die für Studierende gut zugänglich sind, behandeln wir im Unterricht auch Akustikwellen und Wellen auf der Wasseroberfläche. Dabei werden Phänomene betrachtet, welche durch die Wellengleichung für den Schalldruck $p(\vec{r}, t)$ berechnet

$$
\Delta p-\frac{1}{c^{2}} \frac{\partial^{2} p}{\partial t^{2}}=0
$$

wobei $c$ die Schallgeschwindigkeit ist. Dabei werden mit den Ingenieurstudierenden die Zusammenhänge zwischen Frequenz $f$, Wellenlänge $\lambda$ und Schallgeschwindigkeit $c$ diskutiert, in dem die Wellenfunktion $p=p_{0} \sin (x / \lambda-\omega t)$ in die Gleichung eingesetzt wird. Als zusätzlicher Term könnte in (1) die Dämpfung noch berücksichtigt werden, dargestellt als Operator, in dem die Viskosität des Mediums vorkommt. Dies wird aber in den Formeln bewusst weggelassen, auch wenn es in den numerischen Simulationen berücksichtigt wird.

Unter Zuhilfenahme des „Rippeltanks“, eines Experimentes, das in Abb. 4.3 dargestellt ist, kann die Äquivalenz der verschiedenen physikalischen Wellenphänomene gut diskutiert werden. Aus diesem Grund ist das Experiment auch den Namensgeber für die eingesetzte Software (Falstad). Es kann eine sehr gute qualitative Übereinstimmung von Simulation und Messung gezeigt werden. 
Abb.4.3 Experiment zur Darstellung von

Wellenausbreitung und Interferenzmustern. Es wird eine Wanne mit wenig Wasser gefüllt. Die Phänomene werden mit den Oberflächenwellen gezeigt. Mit einer harmonischen Anregung und einer Stroboskoplampe werden die Wellenzüge visualisiert

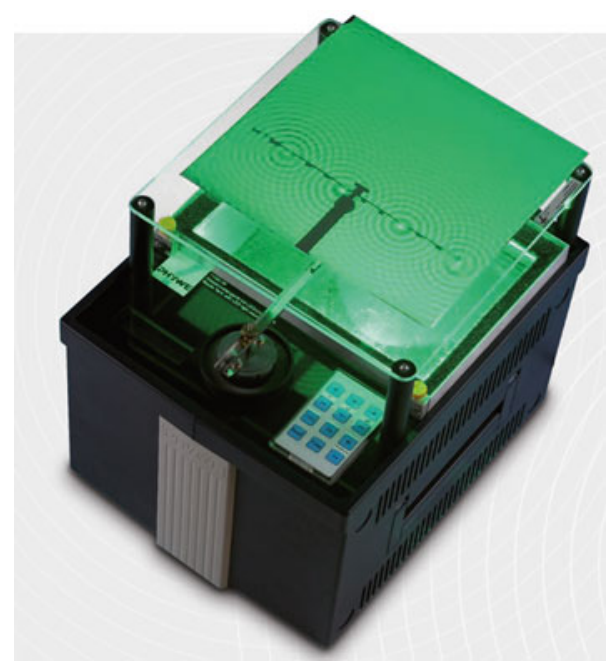

Im „Rippletank“ Softwaretool kann zu Laufzeit hin und her gesprungen werden zwischen den verschiedenen physikalischen Effekten, sodass für Studierende die Universalität der physikalischen Darstellung (3) gut erfahrbar wird: Es wird eine Auswahl zwischen Lichtwellen, Radiowellen in drei verschiedenen technisch relevanten Frequenzbereichen und akustischen Wellen angeboten (siehe Abb. 4.4).

Die Behandlung von Wasserwellen ist weitaus komplizierter als dass sie mit Gleichung (4.3) vollständig beschrieben werden könnte. Ohne auf die numerischen Lösungen einzugehen, wird das Verhalten von Wasserwellen in der Vorlesung im Frontalunterricht behandelt und in den Übungen anhand von Fotografien bearbeitet (siehe Abb. 4.5).

\section{Anschauliche Betrachtung von quantenmechanischen Teilchen als Hinführung zur Halbleiterphysik und Atomphysik}

Die Behandlung der Quantenmechanik an einer Ingenieurschule ist deshalb eine Herausforderung, weil sie traditionell mit einem umfangreichen analytischen Instrumentarium behandelt wird. Darauf wird in diesem Lernobjekt verzichtet. Zweitens - und besonders ausgeprägt, wenn man auf die visuelle Darstellung der Phänomene setzt - stellt sich berechtigterweise die Frage: wo brauche ich dieses Wissen später? Kaum ein Ingenieur lässt einzelne Elektronen durch den Raum 


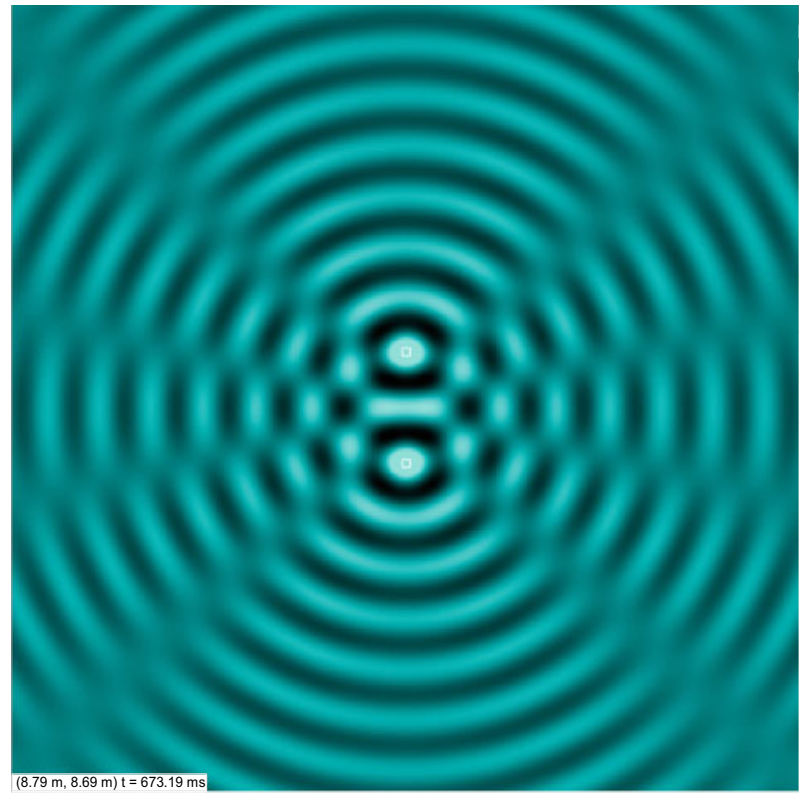

Abb. 4.4 Akustik-Simulation: Überlagerung zweier Sinusquellen. Inlet oben rechts: Auswahlmöglichkeiten für die Interpretation der numerischen Lösung. Links unten werden die Koordinaten des Mauszeigers sowie die fortschreitende Zeit angezeigt. Übungsaufgaben: Wellenlänge auslesen, um daraus die Frequenz zu berechnen, Einzeichnen der Bereiche mit konstruktiver Interferenz und der Orte wo sich die beiden Quellen auslöschen

fliegen oder macht Quantenmessungen. Als Grundlage der Halbleiterphysik hat die Quantenphysik aber trotzdem einen hohen Stellenwert im Curriculum und wird in den Lernzielen entsprechend erwähnt, siehe 4.1.2.

Der in diesem Projekt gewählte Weg wird analog zur Elektrodynamik aufgebaut und nennt die Schrödingergleichung (inklusive des historischen Kontextes), verzichtet dann aber auf jegliche analytische Weiterbearbeitung. Die Studierenden kennen nach der Vorlesung die folgende Darstellung der Schrödingergleichung

$$
\begin{gathered}
i \hbar \frac{\partial}{\partial t} \Psi(\vec{r}, t)=-\frac{\hbar^{2}}{2 m} \Delta \Psi(\vec{r}, t)+U(\vec{r}) \Psi(\vec{r}, t) \\
P_{V}=\iiint_{V}|\Psi|^{2} d V
\end{gathered}
$$




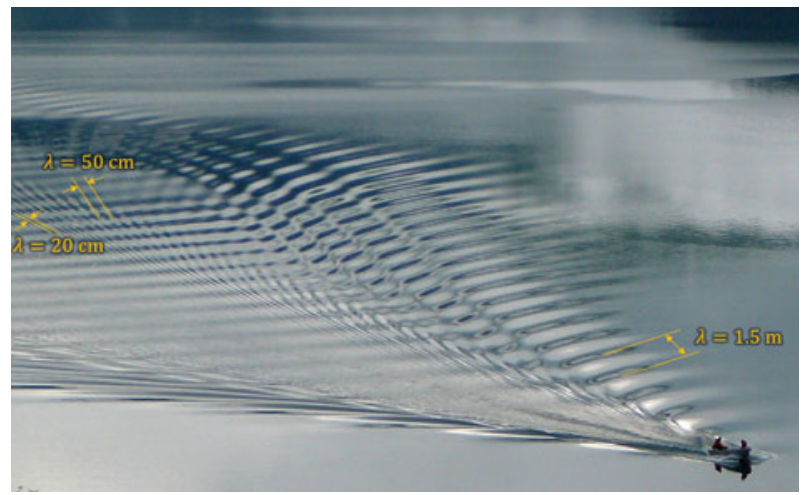

Abb.4.5 Beobachtung der Wellenausbreitung auf der Wasseroberfläche, ausgelöst durch ein fahrendes Boot. Es werden Wellen mit verschiedenen Wellenlängen $\lambda$ erregt. Aufgrund der unterschiedlichen Ausbreitungsgeschwindigkeit je nach Wellenlänge (Dispersion) und der Interferenz entseht ein interessantes Wellenmuster

und können die darin enthaltenen Größen benennen (komplexe Wellenfunktion $\Psi$, reduziertes Planck'sches Wirkungsquantum $\hbar$, Masse $m$, Potenzial $U$ ). Wichtig ist dabei die Aufenthaltswahrscheinlichkeit $P_{V}$, die angibt, wie gross die Chance ist, ein Teilchen in einem vorgegebenen Volumen $V$ anzutreffen. Die Unsicherheit, die damit in der Physik eingeführt wird, führt oft zu Verwirrungen und (populärwissenschaftlichen) Fehlinterpretationen.

Als Grundlage für die Lerneinheit wird ein Simulationstool eingesetzt. Es ist eine für didaktische Zwecke optimierte Quantenmechanik-Simulation ${ }^{3}$, welche im Rahmen dieses Projektes erweitert wurde und ursprünglich aus der schönen Sammlung der Universität von Colorado stammt ${ }^{4}$. Beim Einsatz der Computersimulationen kann auf einige Erkenntnisse aufgebaut werden (Bransford et al. 2002, McKagan, 2010).

Ein wichtiges Element ist die Behandlung der oben erwähnten Unsicherheit, die in der Quantenmechanik schon in den Grundgleichungen anzutreffen ist. Die Software löst die Schrödingergleichung (4) im Zeitbereich mit Wellenpaketen, die im klassischen Bild den „Teilchen“ entsprechen. Als wichtiges Feature hat die Software einen Knopf „Make Quantum Measurement“, der aufgrund der Wahrscheinlichkeitsdichte (die aus der Wellenfunktion $\Psi(\vec{r})$ für jeden Ort berechnet

\footnotetext{
${ }^{3}$ https://github.com/icp-zhaw/icp-quantum-tunneling

${ }^{4}$ http://phet.colorado.edu $\rightarrow$ quantum-tunneling.
} 
werden kann) und mithilfe eines Zufallsgenerators das Teilchen im Simulationsgebiet anzeigt. Was danach passiert, ist ein komplett anderes dynamisches Verhalten des Systems, als wenn die Quantenmessung nicht durchgeführt worden wäre.

Um die Studierenden mit diesen Wahrscheinlichkeiten arbeiten zu lassen, wurde das bestehende Tool $^{5}$ um einige Features erweitert. Dabei wurden verschiedene Aufgabenstellungen möglich, in denen die Studierenden die Materialien wählen können. So sollen sie beispielsweise ein Elektron in einem Quantentopf einsperren oder es durch eine Wand ,hindurchtunneln“ lassen. Letzteres Beispiel wurde in Abb. 4.6 dargestellt.

Beim Umgang mit der Schrödingergleichung ist es in unserer Veranstaltung zentral, die Brücke zu schlagen zu den Konsequenzen, die aus der Quantenmechanik folgen. Einerseits ist dabei die Halbleiterphysik zu nennen mit den wichtigen elektronischen Bauteilen wie Diode, Transistor oder Solarzelle. Dabei ist die in der Simulation eingeübte Anschauung sehr hilfreich: aus der Simulation mit einem Teilchen in einem Potenzialtopf wird auf die Verhältnisse mit mehreren gekoppelten Potenzialtöpfen auf die Situation im Kristallgitter geschlossen. Andererseits ist die Quantenmechanik die physikalische Grundlage der Chemie.

\subsection{Vorgehensweise und Didaktisches Konzept}

Die im vorangehenden Kapitel beschriebenen Lernobjekte werden so aufbereitet, dass sie leicht in einen anderen Kontext übertragen werden und mehrfach wiederverwendet werden können. Die Lernziele sind in den Modulbeschreibungen der Veranstaltungen festgehalten (siehe Abschn. 4.1.2) und werden für das Projekt als Randbedingung angesehen. Das didaktische Konzept und insbesondere das Experimentieren mit modernen didaktischen Methoden ist in einem gewissen Rahmen den Dozierenden überlassen und konnte im Rahmen der laufenden Veranstaltung angepasst und optimiert werden.

\section{Design Based Research und Seamless Learning Ansatz}

Methodisch folgt das Projekt den Prinzipien des Design Based Research (Brown, 1992) und inhaltlich werden die in (Wong \& Looi, 2011) beschriebenen Nahtstellen des Lernens betrachtet. In Zusammenarbeit mit dem Basisprojekt (Dilger

${ }^{5}$ http://phet.colorado.edu $\rightarrow$ quantum-tunneling. 


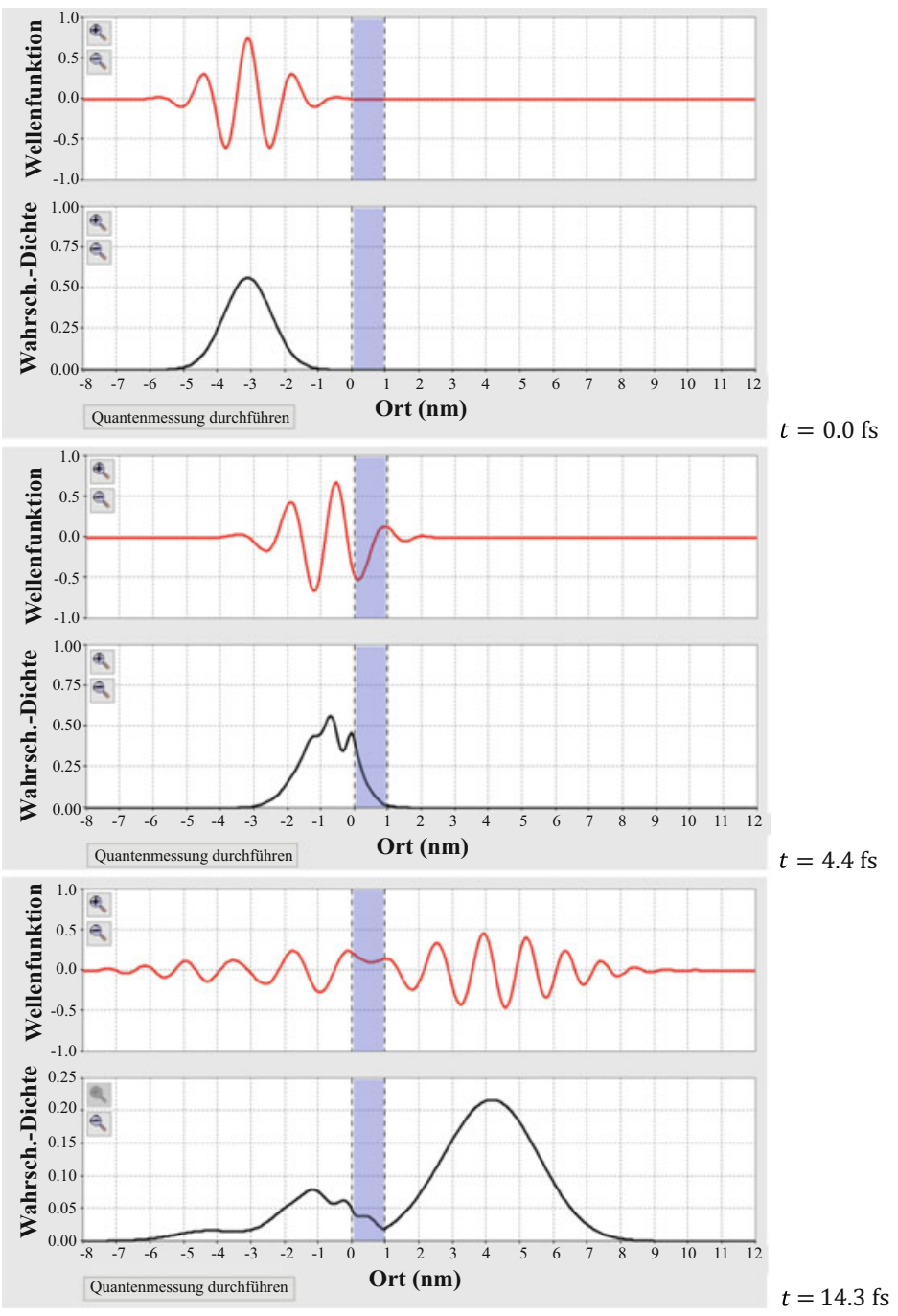

Abb.4.6 Simulation eines nach rechts fliegenden Teilchens. Die blau eingefärbte Zone zwischen $0 \mathrm{~nm}<x<1 \mathrm{~nm}$ ist eine Potenzialbarriere. Das Teilchen dringt teilweise durch die Barriere und wird teilweise reflektiert. Mit dem Knopf „Quantenmessung durchführen“ wird evaluiert, wo das Teilchen sich genau aufhält. Bei der Messung verändert sich die Wellenfunktion 
et al., 2019) des gemeinsamen IBH Labs Seamless Learning ${ }^{6}$ wurde ein didaktisches Konzept erarbeitet und die Lernmaterialien in mehreren Schritten evaluiert und verbessert.

Die Brüche im Sinne des Seamless Learning, die in diesem Projekt besonders relevant sind, können in vier Gruppen eingeteilt werden.

Erstens sind im Physikunterricht die kognitive Herausforderung besonders ausgeprägt. So muss immer wieder der Übergang gemacht werden zwischen der analytischen Beschreibung eines Phänomens (mathematische Formel) und der Beobachtung oder Vorstellung der Zusammenhänge in der realen Welt. Wir sehen in diesem Zusammenhang einen Aufklärungsbedarf, der über das Aufzeigen der Bruchstellen besonders gut angegangen werden kann: Im Gegensatz zu den Studierenden haben Physikdozierende das hin und her springen zwischen der Formelwelt und der realen Welt oft schon jahrelang geübt. Im Frontalunterricht stiftet die gutgemeinte Erwähnung eines Anwendungsbeispiels möglicherweise Verwirrung, da den Studierenden die mentale Übertragung nicht sofort gelingt. Bei einer ad hoc Referenz zu einer Anwendung oder beim Einbinden einer Illustration in einen Vortrag soll kontrolliert werden, ob die Studierenden das Beispiel und den Bezug zu den Formeln verstanden haben. Eine sorgfältige Planung, die diesen Bruchstellen genügend Zeit einräumt, kann diese frustrierenden und verwirrenden Momente in Erfolgserlebnisse wandeln.

Zweitens stellen Nahtstellen in den Lernbiografien Brüche im Sinne des Seamless Learning dar: einerseits mit der Anknüpfung der aktuellen Unterrichtssituation an vorangehende Veranstaltungen oder an Stoff aus der Schulbildung. Andererseits schafft man gerne Referenzen in die Zukunft und erwähnt Anwendungen aus dem mutmaßlichen Berufsalltag nach Abschluss des Studiums. Diese Referenzen - bewusst gewählt und sorgfältig ausgearbeitet - haben ein großes Potenzial zur Erklärung und Motivationssteigerung.

Drittens - speziell ausgeprägt im Zusammenhang mit Computersimulationen und den Aktivitäten auf den mobilen Endgeräten der Studierenden - verbergen sich im Bruch zwischen analogen und digitalen Lernressourcen ähnliche Herausforderungen. In einer naiven Herangehensweise ist man versucht, das analoge Experiment und die Simulation möglichst nahe aneinander zu bringen, so wie in der konkreten Anwendung des Rippeltanks. Analog und digital produzieren dann sehr ähnliche Bilder, was aber bei den Studierenden möglicherweise keinen bleibenden Eindruck hinterlässt. Die Überlegungen, die nötig sind, um die Äquivalenz der Resultate zu diskutieren, tragen nicht unbedingt zum intuitiven Verständnis

\footnotetext{
${ }^{6}$ Seamless Learning: Grenz- und kontextübergreifendes Lehren und Lernen in der Bodenseeregion https://seamless-learning.eu/.
} 
bei. Im Kontrast dazu sollten numerische Simulationen bewusst dort eingesetzt werden, wo keine Experimente möglich sind; und Labormessungen dort, wo die Simulationen zu kompliziert werden. Analoge und Digitale Experimente ergänzen sich also und unterstützen gemeinsam ein umfassendes Verständnis für die physikalischen Phänomene. Auch hier muss der Übergang genügend Beachtung finden, möglicherweise mit etwas Reservezeit in der Planung der Lektionen.

Viertens wurde in diesem Projekt eine zusätzliche Nahtstelle identifiziert, die in der Literatur noch nicht als Seamless Learning Bruch beschrieben wurde, da sie sehr spezifisch für das gewählte Thema ist: Wir erkennen in den Grundgleichungen verschiedener Disziplinen immer wieder die Wellengleichung - eine formalmathematische Übereinstimmung einerseits. In der Beobachtung der Phänomene andererseits gibt es bei der Wellenfortpflanzung interessante Übereinstimmungen, etwa bei der Ausbildung eines Wellenpakets oder wenn sich Interferenzmuster bilden. In der Reflexion der hier geschaffenen Unterrichtsmaterialien rücken wir dieses Erkennen von gleichem Verhalten in verschiedenen Themenbereichen der Physik in den Kontext des Seamless Learning. Über die Grenzen der Themenbereiche hinaus gibt es hier Gemeinsamkeiten. Makroskopische Beobachtungen können so beispielsweise genutzt werden zur Erklärung von quantenmechanischen Phänomenen, oder Vorgänge auf einer den Studierenden zugänglichen Zeitskala können Effekte erklären, die in Realität sehr schnell ablaufen und nicht beobachtet werden können.

\section{Veröffentlichung der Lernobjekte und Übertragung der Resultate auf andere Veranstaltungen}

Während die didaktische Qualität bei der Wahl der Werkzeuge das wichtigste Kriterium war, haben wir bevorzugt Open Source Produkte gewählt. Die Quelloffenen Tools haben den Vorteil, dass sie erweitert werden und günstig weiterverbreitet werden können. Im Fall der Visualisierung von quantenmechanischen Teilchen haben wir eine existierende Software erweitert und zum Ende des Projektes wiederum im offenen Quellcode veröffentlicht ${ }^{7}$. Dabei ist der Mehrwert nicht isoliert in der Software zu sehen, sondern im Gesamtpaket (was wir als „Lernobjekt" bezeichnen), also inklusive dem Foliensatz für den Frontalunterricht, den Fragestellungen und Musterlösungen für das Praktikum. Ergänzend dokumentiert eine Beschreibung für Dozierende die Hintergrundinformationen und Erkenntnissen aus den bisherigen Durchführungen. Das Material wird auf der

${ }^{7}$ https://github.com/icp-zhaw/icp-quantum-tunneling 
gemeinsamen Seamless Learning Plattform https://seamless-learning.htwg-konsta nz.de/ systematisch dargestellt und in den Kontext der anderen Projektergebnisse gebracht.

Zweimal wurden im Rahmen des Projektes die Übertragung der Materialien in eine andere Veranstaltung durchgeführt. Erstens als das Lernobjekt zur Beschreibung der leitungsgebundenen elektromagnetischen Wellen von der Vorlesung Physik 3 in die Erstsemestrigenveranstaltung für Informatiker portiert wurde. Zweitens musste wie in Abschn. 4.1.3 beschrieben im Frühlingssemester 2020 die Präsenzveranstaltung in den Fernunterricht übergeführt werden. In beiden Fällen hat sich die Strukturierung der Lernobjekte und die begleitende Forschung im Rahmen des Design Based Research positiv ausgewirkt. Die Erkenntnisse aus der Durchführung in einem anderen Anforderungsprofil bzw. in einem anderen Format werden im Abschn. 4.4 beschrieben.

\section{Didaktisches Konzept}

Bei der Überarbeitung des didaktischen Konzepts des einsemestrigen Moduls „Physik: Felder und Wellen“ im Studiengang Systemtechnik hat man sich als Startpunkt an den Durchführungen früherer Jahre orientiert und diese weiterentwickelt. Es wird davon ausgegangen, dass sich die Studierenden in der Assessmentstufe bereits für das Ingenieurstudium qualifiziert haben, und deshalb unterstützen wir gerade auch die schwächeren Studierenden mit relativ viel Aufwand, so dass sie dem anspruchsvollen Stoff folgend zu können. Diese Unterstützung findet in der Kontaktzeit in den Halbklassen statt (Übungen/Praktikum). Ein weiterer Kommunikationskanal ist die Lernplattform Moodle.

Aufgrund des gedrängten Stundenplans ist nicht zu erwarten, dass alle Studierenden sich freiwillig die Vorlesung ausreichend vor- und nachbereiten. Die Aktivitäten des Selbststudiums werden deshalb größtenteils angeleitet und die bearbeiteten Aufgaben müssen auf der Lernplattform abgegeben werden. Gruppenarbeiten werden ausdrücklich unterstützt. Es zeigt sich, dass die Studierenden sich den Stoff gegenseitig erklären und bei der gemeinsamen Abgabe nicht den Arbeitsaufwand minimieren.

Das Feedback zu den abgegebenen Resultaten ist wichtig: Zusätzlich zu den direkten schriftlichen Kommentaren in der Lernplattform werden ausgewählte Resultate aus der Lernplattform in die Vorlesung eingebracht. Die an der Gruppenarbeit beteiligten Personen erklären dann ihre Überlegungen, in der darauffolgenden Vorlesung werden die Lösungen allenfalls diskutiert und weiterentwickelt. Somit ist den Studierenden klar, dass sie durch ihre Beteiligung an 
der Gruppenarbeit die abgegebene Lösung auch verstehen und möglicherweise ein paar Tage später kommentieren müssen. Die Bearbeitung der Übung und die Abgabe von Resultaten auf Moodle ist fakultativ, sie werden aber von allen Studierenden genutzt.

Die neuen auf Computersimulationen aufbauenden Lernobjekte fügen sich nahtlos in den Übungsbetrieb ein. Bereits das langjährige Konzept der Vorlesung sieht im Halbklassenunterricht wahlweise Übungen oder Praktika vor, bestehend aus einer Doppellektion mit einer starken Eigenbeteiligung der Studierenden. Die Räumlichkeiten fördern Gruppenarbeiten von zwei bis 4 Personen. Durch das gute Betreuungsverhältnis im Halbklassenunterricht (ein Dozierender auf ca. 16 Studierende) kann ein persönlicher Kontakt und ein Vertrauensverhältnis aufgebaut werden, so dass die Schwelle sehr tief ist, dass Studierende auch in kompletter Verwirrung Hilfe suchen.

Im Semesterplan werden die Computersimulationen in den Übungen schon früh eingeführt. Die Selbstständigkeit bei der Bearbeitung der Übungen wird im Laufe des Semesters laufend erhöht; während am Anfang jede Aktion im Simulationstool vorgegeben ist und Zwischenresultate abgefragt werden, sind die Aufgabenstellungen gegen Ende des Semesters offener und anspruchsvoller.

In der Vorlesung werden vor allem in den ersten Wochen des Semesters viele Experimente aus der Physiksammlung gezeigt (analoge Experimente). Der Übergang zu den Simulationen (digitale Experimente) wird zuerst in der Vorlesung behandelt und dann im Praktikum durch die Studierenden auf ihren eigenen mobilen Geräten (Laptop, Tablet) weitergeführt.

Durch die Umstellung auf Online-Unterricht (siehe Abschn. 4.1.3) wurde eine größere Hausarbeit ausgegeben, welche als letzte Stufe in der immer größeren Selbstständigkeit und der offeneren Fragestellung gesehen werden kann. Dabei hat sich die Zwischenbesprechung als aufwendige, aber sehr fruchtbare Interaktion zwischen Dozierenden und Studierenden bewährt.

Die mehrmalige Evaluation der Veranstaltung hat zu einer Schärfung des Konzepts geführt. Die Lernobjekte wurden mit jeder Iteration besser in den Semesterplan eingebunden und die Verbindung zwischen Vorlesung und Praktikum wurde intensiviert. Die Studierendenbefragung hat die Aufmerksamkeit auf erfolgreiche kleine didaktische Maßnahmen gelenkt, wie zum Beispiel das Formelblatt, das die im Semesterverlauf eingeführten physikalischen Definitionen und Gesetze auf einem Blatt zusammenfasst.

Es hat sich auch gezeigt, dass die in diesem Projekt erarbeiteten Lernobjekte nicht isoliert betrachtet werden sollten, sondern dass bei deren Verwendung eine aktive und bewusste Integration in das didaktische Konzept gemacht werden muss. Das Zusammenspiel zwischen Lehrenden und Lernenden wird bewusst 
offengelassen. Damit wird die Basis einer großen Verbreitung geschaffen. Die Grundannahme, dass kleingranulare Lernobjekte eine höhere Chance zur Wiederverwendung haben, hat sich damit bestätigt, wobei allerdings der Integrationsaufwand in das didaktische Konzept der Veranstaltung nicht unterschätzt werden sollte.

\subsection{Erkenntnisse}

\section{Spezielle Herausforderungen von Wellenphänomenen}

Wellenphänomene haben eine intrinsische Orts- und Zeitabhängigkeit. Diese sollen im Unterricht sorgfältig behandelt werden. Oft wird in der Elektrotechnik mit einer harmonischen Anregung gearbeitet, also mit einem Signal, das bei einer vorgegebenen Frequenz oszilliert. Die Formeln vereinfachen sich dann zwar, aber die Wellenfortpflanzung wird nur noch mathematisch erfassbar. In diesem Projekt wird vorgeschlagen, einen speziellen Fokus auf das Einschaltverhalten zu setzen und die die Fortpflanzung von Wellenpaketen zu betrachten. Nur dann ist das örtlich-zeitliche Verhalten wirklich intuitiv erfassbar.

In der klassischen Behandlung von Wellenphänomenen, die auf der Verwendung von analytischen Formeln beruht, sind Einschaltvorgänge, Wellenpakete und Streuprozesse oft bereits außerhalb der Reichweite der Fertigkeiten der Studierenden. Die Verwendung von Simulationstools als Alternative zu den mathematischen Umformungen ermöglicht eine Behandlung der Wellenphänomene im Ortsund Zeitbereich.

Simulation unterstützt außerdem die konstruktivistische Herangehensweise. Virtuelle Experimente erlauben Untersuchungen auf Zeitskalen, die sonst für Experimente nicht zugänglich sind. Für elektromagnetische Phänomene bewegt man sich dabei in den Mikrosekunden, in der Quantenmechanik untersucht man Vorgänge in den Femtosekunden.

Beispiele:

- Bei der Konstruktion einer Handyantenne zeigt die Simulation, wie die zeitlich variierenden elektrischen und magnetischen Felder sich als Wellenpaket von der Antenne loslösen, sich dann durch den Raum bewegen und von einer Empfangsantenne wieder aufgefangen werden können.

- Beobachtet man die Vorgänge in einem Mikrowellenofen über ein paar Mikrosekunden, so sieht man am Anfang die Wellenausbreitung und dann die Felder der überlagerten stehenden Wellen, die das Gargut letztlich erwärmen. 
- Ein einzelnes quantenmechanisches Teilchen verhält sich anders als wir es in der makroskopischen Welt gewohnt sind. Es gibt die so genannte WellenTeilchen-Dualität, welche als theoretisches Konzept schwierig zu verstehen ist, aber bei einer zeitaufgelösten Beobachtung eines Wellenpakets sichtbar wird. Die Simulation hilft aufzuzeigen, wie man aus dem Gedankenexperiment mit einem Teilchen und einem Potenzialtopf auf die Beschreibung der Halbleiter kommt und dann bis zur Anwendung, wie z. B. der Solarzelle.

\section{Entwicklung der physikalischen Vorstellung}

Durch das enge Betreuungsverhältnis und die vertrauensvolle Umgebung konnte während der Durchführung die kognitive Entwicklung der Studierenden mitverfolgt werden. Im Vergleich zur Ausbildung des dreidimensionalen Vorstellungsvermögens fordern die Wellenphänomene als zusätzliche kognitive Leistung den Umgang mit Bewegungen in den Strukturen. Es ist dabei nicht die Bewegung eines kleinen Objekts in einem Koordinatensystem (wie es im vorangehenden Physikunterricht mit den Gesetzen von Newton behandelt wurden), sondern eine großflächige, nichtlokale Bewegung, wie sie den Wellenphänomenen eigen ist.

Die Studierenden wurden immer wieder dazu aufgefordert, Handskizzen anzufertigen, wobei sich gezeigt hat, dass sie sehr unterschiedlich weit sind in ihrer Vorstellungskraft und ihren zeichnerischen Fertigkeiten. Es ist in diesen Situationen wichtig, die Zwischenresultate nicht zu bewerten, sondern durch aktives Fragen stellen und zurückhaltende Hilfestellungen die Studierenden dazu zu bringen, Miskonzeptionen zu erkennen und ihre Skizzen selbstständig zu verbessern. Der starke Bezug auf die Semesterendprüfung ist dabei teilweise erschwerend. Es muss vermieden werden, dass der bewertende Direktvergleich zwischen den Studierenden die Schwächeren daran hindert, mutig eigene und möglicherweise Fehlerhafte Skizzen zu erstellen. Gute Anweisungen in den Aufgabenstellungen und ein sorgfältiger Umgang mit Handskizzen ermöglichen es, dass Ideen festgehalten und mit Mitstudierenden oder den Dozierenden besprochen werden und dass dabei die Studierenden aktiv ihre Vorstellungskraft erweitern und beispielsweise dabei die Brücke schlagen zwischen der durch eine Formel beschriebene Welle und der Skizze einer Welle. Ein Element zur Unterstützung dieses Prozesses ist es, die elektronischen Werkzeugte in der Aufgabenstellung erst ganz an den Schluss zu stellen. Dabei wird die Lösung zuerst in der eigenen Intuition entworfen und erst danach werden die Handskizzen anhand von der Computersimulation validiert. 
$\mathrm{Zu}$ den verwendeten Simulationstools ist dabei die Behandlung der Wellenphänomene im Zeitbereich essenziell. Für die Studierenden sind Wellenpakete einfacher $\mathrm{zu}$ fassen als eine unendlich ausgedehnte Welle. Im Gegensatz dazu ist die unendlich ausgedehnte Welle, beispielsweise die „Ebene Welle“, als mathematische Formel einfacher darzustellen. Der Übergang zwischen einem immer breiter werdenden Wellenpaket zu einer unendlich ausgedehnten Welle hilft dabei enorm bei der Vorstellung des Konzepts „Ebene Welle“.

Genauso ist der Strahlengang (siehe Abschn. 4.2.1, Abb. 4.1) nur während dem Einschaltvorgang ersichtlich, da sich mit einer eingeschalteten harmonischen Quelle die Wellen überlagern und die Interferenzmuster komplizierter zu interpretieren sind. Auch im Quantum Scattering (siehe Abschn. 4.2.4, Abb. 4.6) sind die Interferenzmuster manchmal verwirrend. Die in diesem Projekt vorgenommene Erweiterung der Simulationssoftware erlaubt die örtliche Integration der Wellenfunktion, interpretierbar als Aufenthaltswahrscheinlichkeit, direkt anzeigen zu lassen.

\section{Akzeptanz bei den Studierenden und Verbesserungen der Lernobjekte bei der Überarbeitung der Prototypen}

Im Rahmen der didaktischen Begleitung wurden Studierendenbefragungen durchgeführt, einerseits per Fragebogen vor und nach dem Einsatz der neuen Unterrichtseinheiten und andererseits durch ein Interview mit der Klasse unter Abwesenheit des Dozierenden. In den Antworten der Studierenden hat sich die vermutete Attraktivität des Einsatzes von Simulationssoftware grundsätzlich bestätigt. Allerdings wurde in den Befragungen während der ersten Durchführung erkannt, dass die Studierenden unsicher waren, wie sich die in den Simulations-Übungen vermittelten Inhalten prüfen lassen. In den Folgeveranstaltungen wurden ganz zu Beginn der Lerneinheiten mit Simulationen schon Beispiele von möglichen Simulationsaufgaben in der Semesterendprüfung gezeigt.

Weiter hat sich in den Studierendenbefragungen gezeigt, dass exploratives Erkunden der physikalischen Zusammenhänge gute Anleitungen braucht und dass die Mehrheit der Studierenden geschlossene Aufgabenstellungen mehr schätzt als Übungsaufgaben mit viel Raum für Kreativität. Das im Projekt angestrebte Experimentieren mit Simulationswerkzeugen hat sich damit als größere Herausforderung erwiesen. Eine erfolgreiche Aufgabenstellung, die zu viel Kreativität und einem explorativen Umgang mit den Tools geführt hat, war die Anweisung, dass sich die Studierenden gegenseitig unter Verwendung der Simulationssoftware Übungsaufgaben gestellt haben. Die Aufgaben wurden in Zweiergruppen 
entworfen, auf die Lernplattform hochgeladen und in der darauffolgenden Woche durch die anderen Gruppen gelöst. Als Dozierender hat man damit genügend Zeit, die Aufgabenstellungen durchzusehen und sie für die nächste Woche freizugeben. Einige Aufgaben waren unlösbar oder zu einfach. Während des Praktikums der Folgewoche haben die Zweierteams die Aufgaben bearbeitet und diskutiert. Während die gut funktionierenden Aufgaben für Peer Instruction geeignet waren, konnten die ungeeigneten Aufgaben im Direktkontakt zwischen Studierenden und Dozierenden nachbesprochen werden. Zusammenfassend kann festgehalten werden, dass die Variation der Sozialform gut funktioniert hat und für Aktivierung der Studierenden und Lernerfolg einen wichtigen Beitrag geleistet hat.

Wir waren erstaunt über die Klarheit, mit der sich die Studierenden möglichst detailliert angeleitete Übungen und geschlossene Fragestellungen wünschen. Das in diesem Projekt angestrebte explorative Lernen war also nicht so beliebt. Die Analyse hat gezeigt, dass es die Studierenden verunsichert und dass die schulisch erfolgreicheren sich eher darauf einlassen (während es als Maßnahme gedacht war, die mathematisch weniger sicheren zu aktivieren). Die kreative Komponente des „schöne Bilder produzieren“, die in der Antragsphase als mögliches Nebenziel der Lernobjekte erwähnt wurde, wurde dann nicht umgesetzt, da sich mit Blick auf die Lernziele nicht gelohnt hätte. Unsere Hypothese ist, dass man diese Aktivitäten nur mit entsprechenden Lockerungsübungen und mit etwas höherem Zeitaufwand durchführen könnte. In der ersten Durchführung mit Studierenden in Systemtechnik wurde es ansatzweise versucht, aber noch ohne Erfolg. In der Durchführung mit Informatikern konnte wegen des engen Stoffplans nicht darauf eingegangen werden. In der zweiten Durchführung in Systemtechnik wurde das Anliegen aufgrund der Umstellung auf Online-Unterricht ebenfalls fallen gelassen.

In der Studierendenbefragung hat sich folgende Überlegung zur Auswahl der Tools geschärft (was auch mit Blick auf die Brüche im Sinne des Seamless Learning Ansatzes schlüssig ist): Einerseits unterstützt ein für die didaktische Visualisierung optimierten Tool die Überwindung des Bruchs zwischen der mathematischen Formulierung und der Anschauung (die Wahl entspricht den in 4.2.1 und 4.2.4 beschriebenen Werkzeugen). Andererseits sind bei Verwendung eines kommerziellen Tools (siehe Abschn. 4.2.2) die Studierenden motivierter, da sie erkennen, dass sie erkennen, dass die Simulationssoftware im späteren Berufsalltag nützlich sein könnte. Dabei wird aber der Bruch Theorie-Anschauung stärker gefordert ist, da die Software beispielsweise die Wellen nicht in Bewegung zeigt, sondern nur als statische Kurvenschaf auf dem Bildschirm abbildet. 


\section{Gewinnbringende Kombination analoger und digitaler Experimente}

Die auf Simulation basierenden Lernobjekte werden in der Ingenieurausbildung an der School of Engineering der ZHAW als Ergänzung zu den im Hörsaal durchgeführten Experimenten gesehen. Im Hands-On Praktikum machen die Studierenden sowohl Laboruntersuchungen an realen Objekten als auch Computerexperimente. Als Lehrperson beobachtet man mit Sorge das Ablenkungspotenzial, das bei Arbeiten am Bildschirm von den digitalen Tools ausgeht. Hier kann es helfen, wenn die Methodik bewusst gemacht wird und von der Erfahrung mit analogen Physikexperimenten (z. B. wird anhand einer Messreihe ein funktionaler physikalischer Zusammenhang ermittelt) in den digitalen Kontext übertragen wird (auch hier muss man oft repetitiv und systematisch die Eingangsgrößen neu wählen und eine numerisch ermittelte Ausgangsgröße manuell erfassen).

Die letzte Durchführung im Frühlingssemester 2020 musste aufgrund der Covid-19 Pandemie größtenteils ins Online-Teaching Format übergeführt werden. Grundsätzlich hat sich dabei bestätigt, dass die Simulationsübungen eine effektive Möglichkeit darstellen, wie die Studierenden auch im Fernunterricht aktiviert werden können. Es hat sich aber auch gezeigt, dass das Fehlen der physischen Hardwareexperimente ein Verlust ist. Für eine Präsenzhochschule ist ein guter Mix aus analogen und digitalen Ressourcen das Optimum.

\section{Transfer der Materialien in andere Anlässe}

In der ursprünglichen Fragestellung wurde angenommen, dass die erarbeiteten Lernmaterialien auch für Lernende in den Schulstufen der Grundschule (Unterrichtsbereiche Natur und Technik) und des Gymnasiums (Physikunterricht) anwendbar sind. Es hat sich gezeigt, dass die für die Fachhochschule ausgearbeiteten Materialien zwar den Ingenieurstudierenden einen einfachen Zugang zu den Phänomenen erlauben, dass aber der Graben für einen Transfer in die unteren Schulstufen weitausgrösser ist als ursprünglich angenommen. Dabei sind einerseits die Fragestellungen nicht dem aktuellen Curriculum angepasst und andererseits müssen die Lehrpersonen für die Überführung der Materialien einen signifikanten Aufwand leisten. Als sich gezeigt hat, dass die ursprünglich geplante Anwendung der Materialien innerhalb der Projektlaufzeit nicht bewerkstelligt werden konnte, hat man die Fragestellung im Projekt abgeändert und die vielversprechendere Transferleistung von Systemtechnik-Studierenden im dritten Semester auf Informatik-Studierende im ersten Semester ins Auge gefasst. Es hat 
sich bewährt, dass die Lernmaterialien in kleinen Einheiten strukturiert wurden (die so genannten „Lernobjekte“), so dass auch vom vorgegebenen Curriculum eine entsprechende Passung gefunden werden konnte.

Als Resultat lässt sich festhalten, dass der Umgang mit Simulationen im Physikunterricht sich auch in der neuen Zielgruppe bewährt hat, dass jedoch der Transfer über mehrere Schulstufen ein zu hohes Ziel war. Dabei hatten die Informatikstudierenden wesentlich weniger relevantes Vorwissen als ihre Kommilitonen in den höheren Semestern im Studiengang Systemtechnik. Es wurde das in Abschn. 4.2.2 beschriebene kommerzielle Tool Tina genutzt, um die für die Informatik wichtigen leitungsgebundenen Wellen darzustellen. Trotz der etwas tieferen intrinsischen Motivation für Physik bei Informatikern konnte mit dem explorativen Lernen anhand von Simulationen die Aktivität der Studierenden hochgehalten werden.

In den Fragestellungen des Projektes wurde die Hypothese aufgestellt, dass moderne elektronische Endgeräte wie die verschiedenen Tablet Devices mit Touchscreen, den Umgang mit den Simulationen verändert. Ein solcher Effekt konnte in unserem Projekt nicht beobachtet werden. Auch die Simulation mit elektromagnetischen Wellen, wo mit dem Finger auf dem Touchscreen Wellen ausgelöst werden können, konnten keine Aufgabenstellungen gefunden werden, die das Experimentieren gefördert hätten. Der mögliche künstlerische-kreative Anspruch, der mit den Tools schöne Bilder produziert und explorativ die Grenzen der technischen Nützlichkeit sprengt, bräuchte mehr Freiräume als wir im vorliegenden Projekt anbieten konnten. Im Kontext des Ingenieurstudiums waren die konkreten technischen Fragestellungen, die letztlich mit Tastatur und Maus besser bearbeitet werden konnten, erfolgreicher. Möglicherweise ist auf einer tieferen Schulstufe das taktile Element wichtiger. Auch wenn die Endgeräte der Studierenden sich bezüglich Alter und Leistungsfähigkeit stark unterschieden hatten, waren die Studierenden mit einem Laptop ohne Touchscreen oder einem leistungsschwächeren Gerät nie im Nachteil.

\subsection{Fazit}

Die Vermutung hat sich bestätigt, dass der Einsatz von Simulationssoftware im Unterricht - speziell für das Thema Wellenausbreitung - einen positiven Effekt auf die Motivation der Studierenden und den Lernerfolg hat. Die positive Einstellung der Studierenden gegenüber den Lerninhalten wurde mehrfach in den Befragungen bestätigt. Für Themen wie die Quantenmechanik, wo Versuche und 
Labormessungen oft gar nicht möglich sind, ist dies ein wichtiger Beitrag zum Lernerfolg.

Die visuelle Vorstellungskraft der Studierenden war anfangs unterschiedlich stark ausgebildet und die Wellensimulationen haben geholfen, die anspruchsvollen Konzepte der elektromagnetischen Felder und quantenmechanischen Teilchen zu erfahren.

Wichtige Vorgehensweisen aus dem Ingenieuralltag konnten mithilfe der verwendeten Softwareanwendungen vermittelt werden: Die Modellbildung am Anfang entspricht dem Erfassen der Phänomene und deren Formulierung als mathematische Formeln. Die Diskretisierung und Simulation auf dem Computer erlaub eine weitere Behandlung der Phänomene nahe an der Realität, ohne die Begrenzung auf Spezialfälle und ohne mathematische Umformungen. Entsprechend der eigenen Vorstellungskraft wird oft eine Skizze der erwarteten Lösung erstellt, bevor simuliert wird, dann wird anhand der numerischen Lösung überprüft, ob die Hypothese korrekt ist. Die Auswertung der Simulationsresultate hat einen hohen Stellenwert und erhält in der vorgestellten Vorgehensweise ein angemessenes Gewicht.

Die neu erschaffenen Lernobjekte basieren teilweise auf bestehenden Simulationstools, die für den Unterricht optimiert wurden. In einem Fall wurde ein kommerzielles Entwurfswerkzeug eingesetzt, was von den Studierenden insofern geschätzt wurde, da sie dieses später möglicherweise im Berufsalltag wieder antreffen werden. Das Material wurde gemäß dem Design Based Research Prozess entworfen und optimiert. Die Kommunikation mit den Studierenden konnte von Durchführung zu Durchführung ebenfalls verbessert werden. Konkret konnte die ursprünglich geäußerte Angst vor der Prüfung - insbesondere in Bezug auf die durch Simulation abgedeckten Lerninhalte - wesentlich reduziert werden. Damit wurde die Prüfungsvorbereitung effizienter und die Motivation verbessert.

Die unterschiedliche Durchführung des Praktikums, einmal vor Ort (erster Prototyp) und dann ein Jahr im Fernunterricht (überarbeitetes Material) erschwert leider den Direktvergleich, eröffnet aber die Chance, den Einsatz von Computersimulation im Onlineunterricht zu beurteilen. Die mit dem Design Based Research Ansatz in mehreren Iterationen optimierten Lernobjekte konnten klar verbessert werden und die erwarteten positiven Impulse im Fernunterricht wurden bestätigt.

Die Weiterführung der verschiedenen Veranstaltungen, in denen die erarbeiteten Lernobjekte zum Einsatz kommen, bringt eine weitere inkrementelle Verbesserung mit sich. Die Publikation der Materialien und die didaktische Aufarbeitung im Seamless Learning Kontext und der aufgezeigte Transfer in eine andere Veranstaltung führen außerdem $\mathrm{zu}$ einer weiteren Verbreitung der Projektresultate. 


\section{Literatur}

Boardman, A. D., Cooper, G. S., \& Swage, J. (1989). The place of computers in the teaching of physics. European Journal of Physics, 10, 161-172.

Finkelstein, N. D. et al. (2005). When learning about the real world is better done virtually: A study of substituting computer simulations for laboratory equipment. Physical Review Special Topics - Physics Education Research, 1, 010103.

Perkins, K., Adams, W., Dubson, M., Finkelstein, N., Reid, S., LeMaster, R., \& Wieman, C. (January 2006). PhET: Interactive simulations for teaching and learning physics. The Physics Teacher, 44. https://www.researchgate.net/publication/241167894_PhET_Interac tive_Simulations_for_Teaching_and_Learning_Physics.

Yee, K. S. (May 1966). Numerical solution of initial boundary value problems involving Maxwell's equations in isotropic media. IEEE Transactions on Antennas and Propagation, 14(3), 302-307.

Kozhevnikov, V. (January 2014). Two-dimensional finite-difference time-domain code with total field/scattered field interface and uniaxial perfectly matched layer absorbing boundaries. Published on Mathworks Contributiones. https://www.mathworks.com/matlabcen tral/fileexchange/41518-2d-fdtd-code-with-tf-sf-interface-and-upml-absorbing-borders.

Falstad, P. (2002). Ripple tank simulation that demonstrates wave motion, interference, diffraction, refraction Dippler effect, etc. http://www.falstad.com/ripple/.

McKagan, S. (Juni 2010). Quantum tunneling PhET Tips for teachers, PDF available from the phet webpage after registration.

Bransford, J. D., Brown, A. L., \& Cocking, R. R. (Hrsg.). (2002). How people learn. Academic.

Brown, A. L. (1992). Design experiments: Theoretical and methodological challenges in creating complex interventions in classroom settings. The Journal of the Learning Sciences, 2, 141-178.

Wong, L. H., \& Looi, C. K. (2011). What seams do we remove in mobile-assisted seamless learning? A critical review of the literature. Computers \& Education, 57(4), 2364-2381.

Dilger, B., Gommers, L., \& Rapp, C. (2019). The learning problems behind the seams in seamless learning. In C. -K. Looi, L. -H. Wong, C. Glahn, \& S. Cai (Hrsg.), Seamless learning: Perspectives, challenges and opportunities (S. 29-51). https://doi.org/10.1007/ 978-981-13-3071-1_2.

Clark, R. C., \& Mayer, R. E. (2003). e-Learning and the science of instruction: Proven guidelines for consumers and designers of multimedia learning. Pfeiffer.

Mazur, E. (1997). Peer instruction: A User's manual series in educational innovation. Prentice Hall.

Föß1, T. (2014). Seamless Learning: Eine Feldstudie über den Einsatz von problembasierten Lernvideos in einem offenen Mathematikunterricht. In M. Ebner \& S. Sandra (Hrsg.), Internet-Technologie und Gesellschaft, Band 5. 
Open Access Dieses Kapitel wird unter der Creative Commons Namensnennung 4.0 International Lizenz (http://creativecommons.org/licenses/by/4.0/deed.de) veröffentlicht, welche die Nutzung, Vervielfältigung, Bearbeitung, Verbreitung und Wiedergabe in jeglichem Medium und Format erlaubt, sofern Sie den/die ursprünglichen Autor(en) und die Quelle ordnungsgemäß nennen, einen Link zur Creative Commons Lizenz beifügen und angeben, ob Änderungen vorgenommen wurden.

Die in diesem Kapitel enthaltenen Bilder und sonstiges Drittmaterial unterliegen ebenfalls der genannten Creative Commons Lizenz, sofern sich aus der Abbildungslegende nichts anderes ergibt. Sofern das betreffende Material nicht unter der genannten Creative Commons Lizenz steht und die betreffende Handlung nicht nach gesetzlichen Vorschriften erlaubt ist, ist für die oben aufgeführten Weiterverwendungen des Materials die Einwilligung des jeweiligen Rechteinhabers einzuholen. 